\title{
Proton beam emittance growth in the Relativistic Heavy Ion Collider
}

\author{
S. Y. Zhang* and V. Ptitsyn \\ Brookhaven National Laboratory, Upton, New York 11973, USA
}

(Received 13 November 2007; published 12 May 2008)

\begin{abstract}
With the significant beam intensity improvement in RHIC polarized proton run 2005 and run 2006, the emittance growth becomes a luminosity limiting factor. The beam emittance growth has a dependence on the dynamic pressure rise, which in RHIC proton runs is mainly caused by the electron cloud. The dependence of the emittance growth on other electron cloud related parameters is also identified. The beam instability is usually absent, and the emittance growth rate is much slower than the ones typically caused by the head-tail instability. It is suspected that the emittance growth is caused by the electron cloud below the instability threshold. A discussion follows.
\end{abstract}

DOI: 10.1103/PhysRevSTAB.11.051001

PACS numbers: $29.20 . \mathrm{db}, 29.27 . \mathrm{Bd}$

\section{INTRODUCTION}

The Relativistic Heavy Ion Collider (RHIC) consists of two rings, named Blue and Yellow. RHIC is designed for heavy ion operations, such as with deuteron, copper, and gold ions, but it is also capable of providing polarized proton collisions. Main machine and beam parameters relevant to past polarized proton runs are shown in Table I.

The application of $200 \mathrm{~m}$ nonevaporable getter (NEG) coated beam pipe in 2004 helped to reduce the highest dynamic pressure rise in long straight sections of both Blue and Yellow rings in RHIC [1]. It allowed one to increase the number of bunches as well as the bunch intensity during the run 2005 operation, where up to 110 bunches with $0.9 \times 10^{11}$ protons per bunch were achieved, compared with 55 bunches with $0.7 \times 10^{11}$ protons per bunch used in run 2004. At the increased beam intensities, the emittance growth was observed. It resulted in less than expected luminosity seen on the physics fills with the highest beam intensities. The intensity threshold of this emittance growth was at approximately $140 \times 10^{11}$ protons in total for the two beams. With additional $180 \mathrm{~m} \mathrm{NEG}$ pipes installed and also due to some changes of the machine configuration, the intensity threshold of the beam emittance growth in run 2006 was increased to $260 \times 10^{11}$ protons in total. The peak luminosity in runs 2005 and 2006 was increased from previous years by factors 2 and 3 , respectively. These are shown in Table II. We note that the application of NEG pipes and related with it the vacuum improvements and the reduction of electron cloud were not the only factors in these luminosity increases. For instance, the reduced number of collision points from run 2005 to run 2006 is also relevant to the improvements.

Throughout this paper, if not mentioned otherwise, the RHIC proton beam emittance data were extracted using the zero degree calorimeter (ZDC) coincident rates measured at the two major experiments, i.e., PHENIX and STAR. During the RHIC runs those measurements were calibrated

\footnotetext{
*syzhang@BNL.GOV
}

with several vernier scans done at the corresponding collision points. The data from other emittance measuring devices available at RHIC, such as the ionization profile monitor (IPM), and the polarimeter target (thin carbon film) scan, were used for verification and comparison, but considered less reliable for accurate absolute values of the emittance. For the beam emittance calculation from the ZDC coincident rates, it is not possible to identify separately the horizontal and vertical emittances as well as separate the emittance data for Blue and Yellow beams. However, from numerous vernier scans, the beam at the store was always found to be round, i.e., the beam sizes and the corresponding emittances on the horizontal and vertical directions were very similar. Usually the difference of the emittances of the two beams is small. We also should mention that all emittance data, presented in this paper, corresponds to the $95 \%$ normalized beam emittance.

The 95\% normalized emittance at the early store (1.5 hour after the ramp event) in runs 2005 and 2006 is plotted against the total beam intensity of each beam in Fig. 1.

The beam emittance growth observed at RHIC proton runs 2005 and 2006 has a dependence on the dynamic pressure rise. For example, the fills with comparable low emittance at early store in both 2005 and 2006 all have comparable low dynamic pressure rise, in spite of the fact that the total beam intensity in 2006 is much higher than that in 2005.

TABLE I. Main machine and beam parameters relevant to past proton runs at RHIC.

\begin{tabular}{lr}
\hline \hline & RHIC \\
\hline Circumference, m & 3834 \\
Number of rings & 2 \\
Kinetic energy at store, GeV & 100 \\
Full bunch length at store, $\mathrm{ns}$ & 14 \\
Average beam size at store, $\mathrm{mm}$ & 4 \\
Number of bunches & up to 110 \\
\hline
\end{tabular}


TABLE II. RHIC polarized proton runs in 2004, 2005, and 2006.

\begin{tabular}{lccc}
\hline \hline \multicolumn{1}{c}{ Run year } & 2004 & 2005 & 2006 \\
\hline Peak luminosity, $10^{30} / \mathrm{cm}^{2} \mathrm{~s}$ & 6 & 11 & 35 \\
Bunch number & 55 & $55-110$ & 110 \\
Bunch spacing, ns & 216 & $216-108$ & 108 \\
Bunch intensity, $10^{11}$ & 0.7 & 0.9 & 1.3 \\
NEG pipes, m & 50 & 250 & 430 \\
\hline \hline
\end{tabular}

The RHIC dynamic pressure rise takes place mainly in warm straight sections (24 single beam long straight sections of 34 meters each and 6 interaction regions of 17 meters each). The dynamic pressure rise is peaked at the end of the beam injection and the beginning of the acceleration ramp. After that it subsides and becomes virtually nonexistent for most of the time during long stores.

The dynamic pressure rise in RHIC proton runs is mainly due to the electron cloud [2,3], which is, therefore, suspected to be a source of the emittance growth. Indeed, the emittance growth at the injection and early ramp shows a dependence on the parameters relevant to the electron cloud, such as the bunch spacing and the bunch length. As a comparison, the emittance growth at the store, where not much of the electron cloud is present, has no dependence on the bunch spacing, but has a dependence on the beambeam parameter instead.

Emittance increases caused by beam instabilities have been often observed in the RHIC operation and beam experiments. It is characterized by a fast growth time (in the order of milliseconds) and can be cured by a proper choice of the machine chromaticities and by the applica-

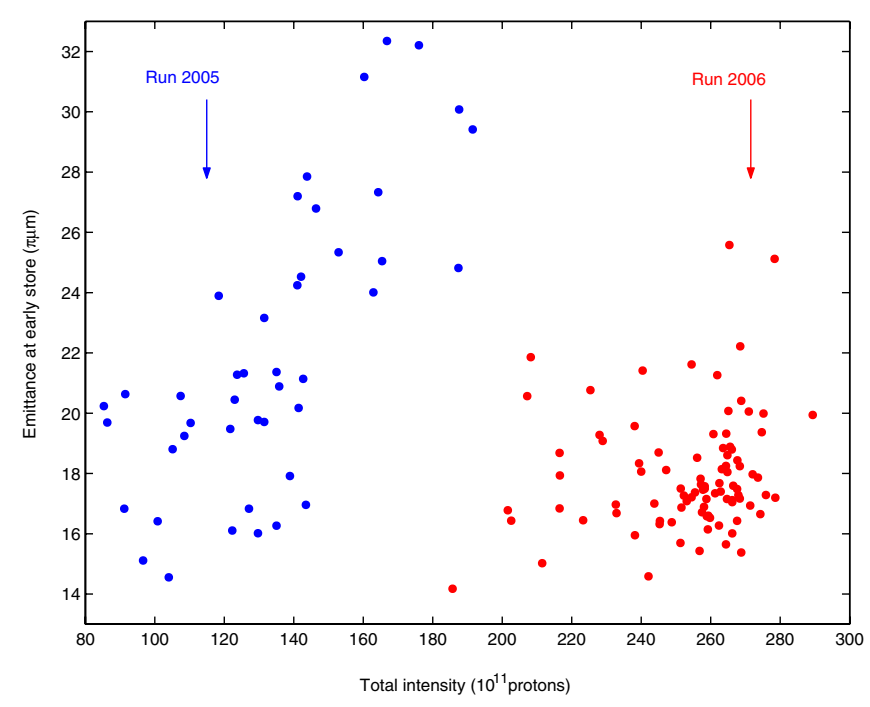

FIG. 1. (Color) The 95\% normalized beam emittance versus total beam intensity for different RHIC fills in 2005 and 2006 $100 \mathrm{GeV}$ polarized proton runs. The emittance is calculated from the average ZDC coincident rates of the experiments PHENIX and STAR, at the early store. tion of octupole correctors. In the latest RHIC heavy ion runs, the effect of the electron cloud on the threshold of the fast transverse instability at the transition crossing was observed, where bunches at the later part of the bunch train were affected by the instability and had increased emittances and bunch intensity losses. This effect presently limits total intensity of beams in the heavy ion operation. Unlike this, the effect of electron cloud on the instabilities in the RHIC operation with protons has not yet been observed. The proton beam emittance growth discussed in this article has a much slower rate, and therefore, it is not believed due to a beam instability. For example, a typical rate for the emittance growth discussed in this article is of the order of $2 \pi \mu \mathrm{m}$ per hour in run 2006. During beam studies, when much higher beam intensity compared with the operation was used, the emittance growth rate achieved about $40 \pi \mu \mathrm{m}$ per hour. Both are much slower than the emittance growth due to head-tail instabilities.

It is suspected that the RHIC proton beam emittance growth is caused by the electron cloud below the head-tail instability threshold. The electron densities in RHIC rings can be calculated from the dynamic pressure rise measured by vacuum gauges, with considerations of the effective vacuum pumping speed, the molecular species, and the electron desorptions. At several straight sections the calculated electron density is of the order of $10^{11} / \mathrm{m}^{3}$ for the high intensity beam, e.g., 110 bunches with more than $1.5 \times 10^{11}$ protons per bunch. This electron density is similar to the electron densities observed in other machines [4]. Since the electron multipacting is very weak or even absent in most ring sections, the average electron density in the entire ring is only of the order of $10^{10} / \mathrm{m}^{3}$ at highest beam intensities. Under the normal operation conditions the average electron density is around $10^{9} / \mathrm{m}^{3}$.

In this article, we present evidences of the RHIC proton beam emittance growth with respect to dynamic pressure rise in operations and beam experiments as well. The correlation of the emittance growth and several parameters relevant to electron cloud is shown, and the corresponding electron densities in RHIC rings at the time are presented. A discussion follows.

\section{DEPENDENCE OF THE EMITTANCE GROWTH ON THE DYNAMIC PRESSURE RISE}

The clear dependence of the beam emittance growth on the dynamic pressure rise was observed in RHIC polarized

TABLE III. Comparison of the fills 7264,7327 in run 2005, and the fill 7909 in run 2006. The emittance is at the early store (1.5 hours after the ramp event).

\begin{tabular}{lrrr}
\hline \hline \multicolumn{1}{c}{ Fill number } & 7264 & 7327 & 7909 \\
\hline Total beam intensity, $10^{11}$ & 143.5 & 187.4 & 268.8 \\
Emittance, $\pi \mu \mathrm{m}$ & 16.9 & 24.8 & 15.4 \\
\hline \hline
\end{tabular}



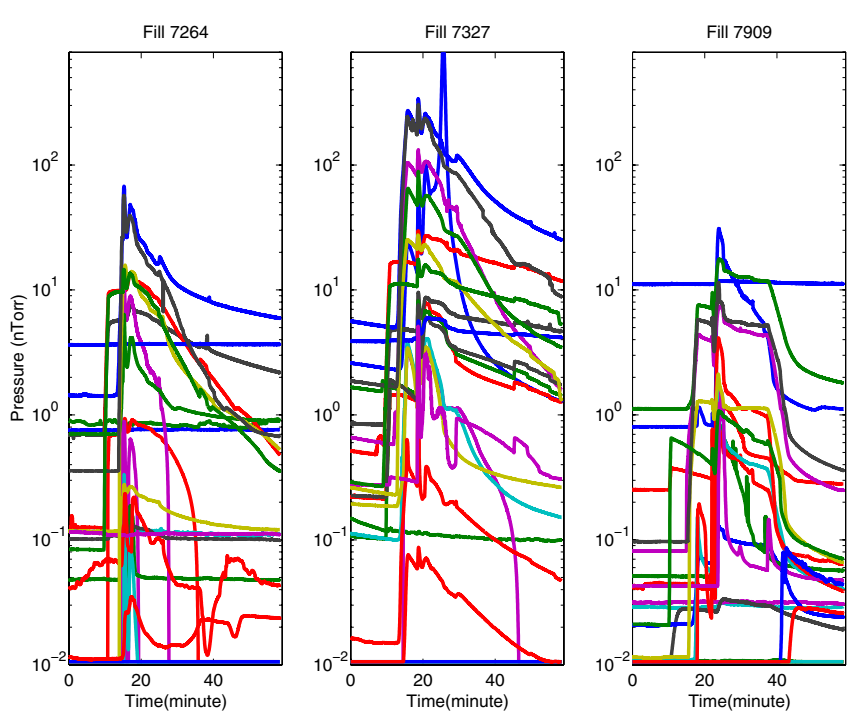

FIG. 2. (Color) The pressure rise in 24 single beam long straight sections in RHIC during the beam injection and acceleration, for the fills 7264 and 7327 in run 2005, and the fill 7909 in run 2006.

proton runs 2005 and 2006. All fills with a small emittance growth had low pressure rise, and, on the other side, all fills with high pressure rise experienced a large emittance growth.

This is illustrated by the fills 7264 and 7327 in run 2005, and the fill 7909 in run 2006. The fill 7327 has a higher intensity, and hence a larger emittance, than 7264 . The fill 7909 in 2006 has a higher intensity, but a smaller emittance than 7327. These are shown in Table III.

The vacuum chamber condition was the same for the fills 7264 and 7327 in run 2005, but it was improved for the fill 7909 in run 2006. The pressure rises measured at the middle of all 24 single beam long straight sections during the injection and the acceleration are shown in Fig. 2 for these fills. As shown, the overall pressure rise is the largest for the fill 7327, and it is the lowest for the fill 7909 .

One may conclude, therefore, the beam emittance at early store has a dependence on the dynamic pressure rise, rather then the beam intensity.
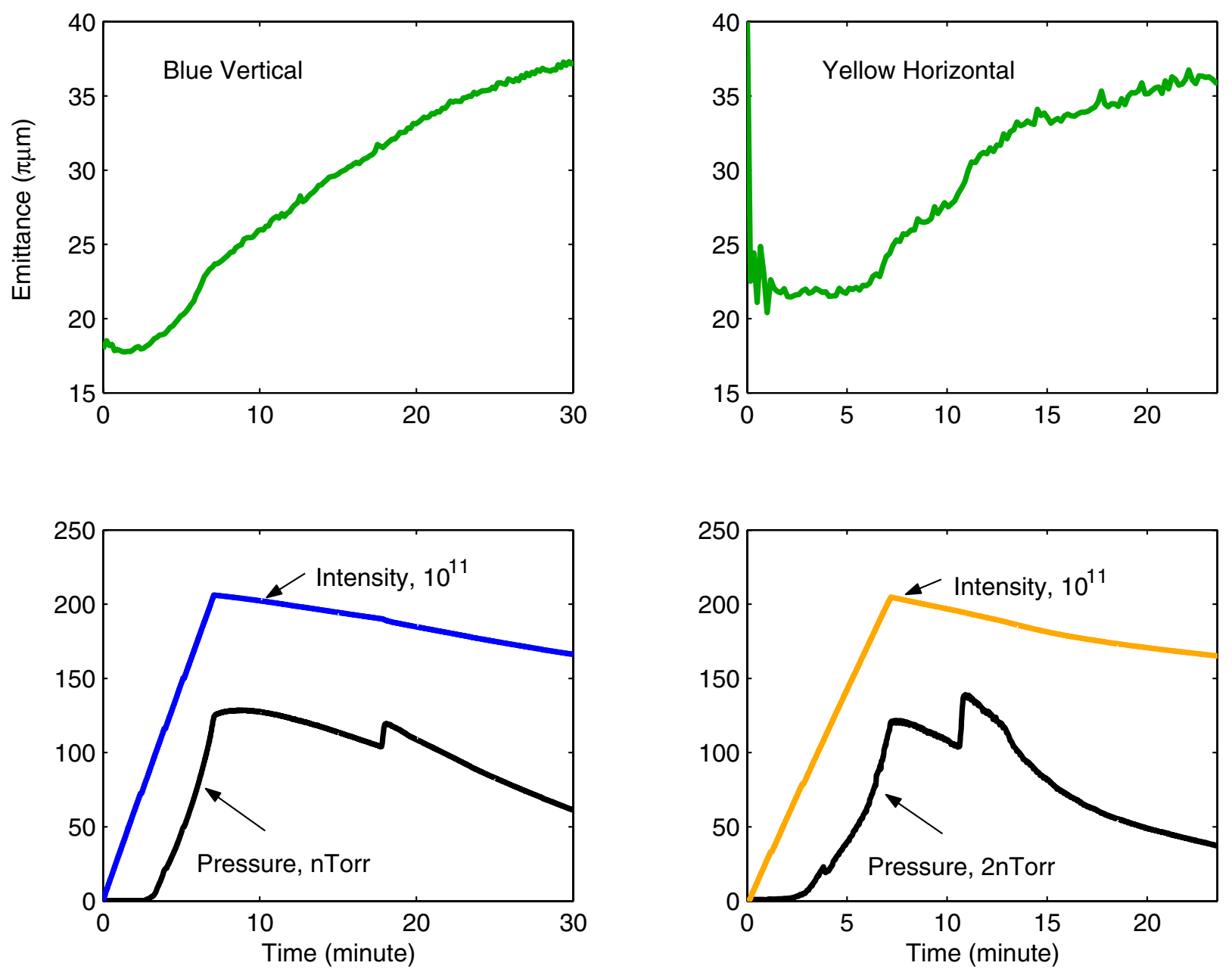

FIG. 3. (Color) Blue ring vertical and Yellow ring horizontal emittance growth observed by IPM for high intensity beams injection, followed by increasing rf voltage. The vacuum pressure is the average taken over 4 long straight sections with highest pressure rise in given rings. 
We note that the emittance of the beam coming to RHIC from the injector chain is relatively constant, and the variation of the beam emittance measured at the early store, shown in Fig. 1, is determined by the emittance growth during the injection and at the beginning of the acceleration ramp. The dynamic pressure rise, shown in Fig. 2, is peaked around that period of time.

In order to explore the dependence of the emittance growth on the dynamic pressure rise in a more direct way, a dedicated beam experiment with a high intensity beam was performed. During the experiment a beam consisting of 110 bunches with $1.9 \times 10^{11}$ protons per bunch was injected, where the bunch intensity was about $50 \%$ higher than used in the RHIC operation. After the injection, the rf voltage was increased from 160 to $300 \mathrm{kV}$ to shorten the bunch length. The shortening of the bunch length would increase the beam peak current, and hence would enhance the electron multipacting. The beam emittance growth observed by the IPM showed correlations with the pressure rise.

In Fig. 3, the Blue ring vertical and Yellow ring horizontal IPM emittance data are shown together with the pressure rises and beam intensities during the experiment. The plotted vacuum pressure is the average taken over 4 long straight sections with the highest pressure rise in given rings. The first increase of the vacuum pressure happens during the beam injection, and the second, smaller, increase corresponds to the rising of rf voltages. Both peaks of the dynamic pressure rise are coincident with the faster emittance growth.

The emittance measurement from the IPM was verified by using the polarimeter target scan to measure the Blue ring horizontal emittance. Figure 4 shows the similar emittance growth measured by both emittance measurement

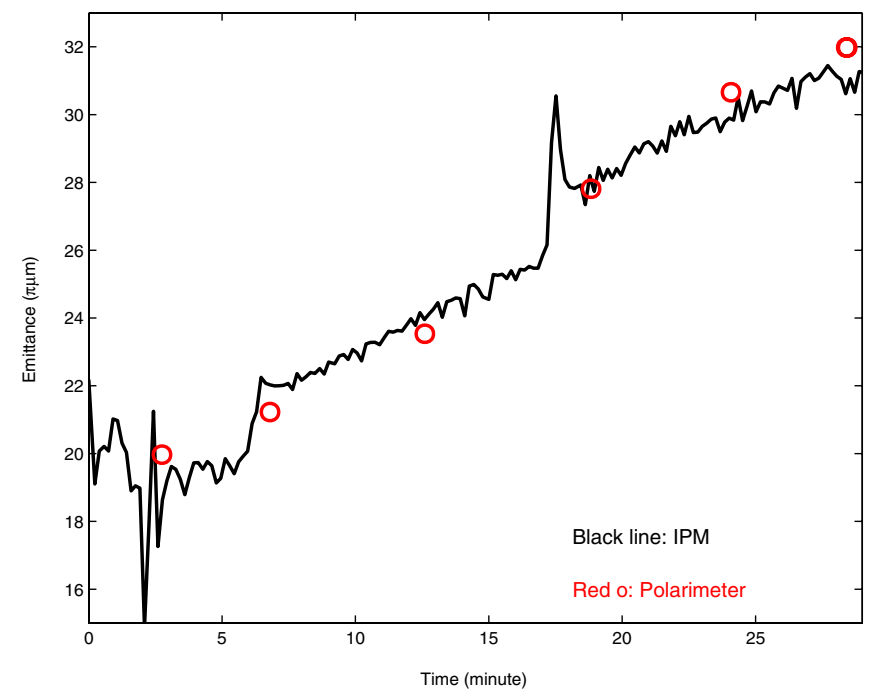

FIG. 4. (Color) Beam emittance growth observed by the IPM for the Blue ring horizontal emittance agrees with the measurement made using the polarimeter target scan.

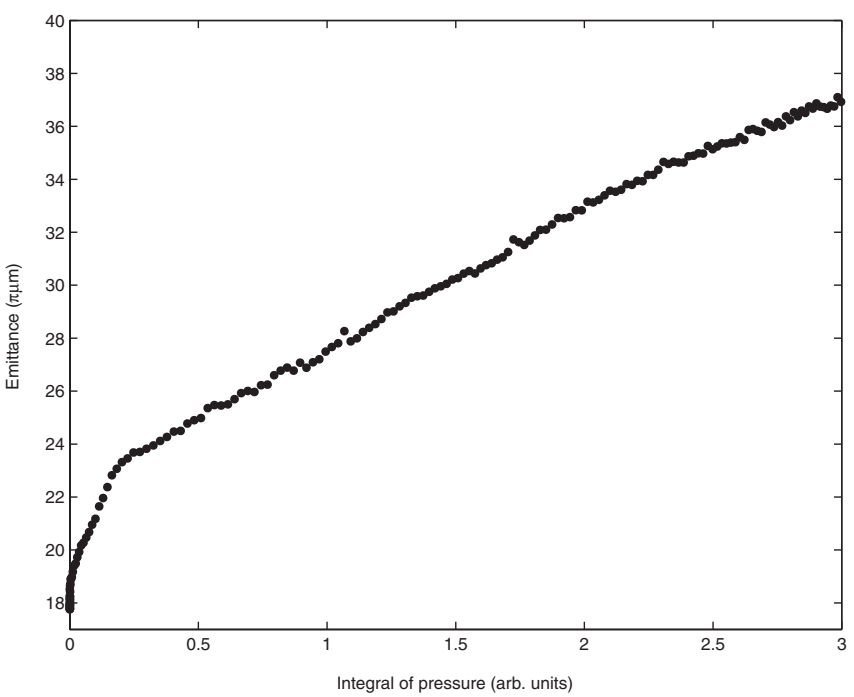

FIG. 5. Blue beam emittance observed by the IPM versus the integral of the dynamic pressure rise at the Blue ring warm section 1.

techniques. Faster emittance increase on 18th-19th minutes happens after the rf voltage rise.

In Fig. 5, the Blue ring vertical emittance measured by using the IPM is plotted against the integral of the pressure rise measured at the Blue ring warm section 1. The relation appears to be linear, except the faster emittance growth at the beginning of the dynamic pressure rise buildup. This might be explained by the delayed establishment of the equilibrium vacuum pressures in the beam chambers. From the linear dependence of the emittance on the integral of the vacuum pressure, one may conclude about similar linear dependence of the emittance growth rate on the dynamic vacuum pressure rise.

\section{DEPENDENCE OF THE EMITTANCE GROWTH ON OTHER ELECTRON CLOUD RELATED PARAMETERS}

\section{A. Dependence on the bunch spacing}

During run 2005, the number of bunches injected into RHIC was gradually increased from 55 to 110 , correspondingly the bunch spacing was reduced from 216 to $108 \mathrm{~ns}$. The bunch intensity in these fills varied between $0.61 \times$ $10^{11}$ and $0.91 \times 10^{11}$ protons. This provided an opportunity to observe the effect of bunch spacing, which is one of the most important electron cloud related beam parameters, on the beam emittance growth. Indeed the effect has been observed, and it is presented in Fig. 6 as the dependence of the measured emittance on the average bunch spacing. The threshold for the emittance growth occurs at an average bunch spacing of approximately $150 \mathrm{~ns}$. For the fills with the average bunch spacing less than $120 \mathrm{~ns}$, the emittance growth is clearly the largest, even that some of those fills had bunch intensities less than $0.75 \times 10^{11}$ pro- 


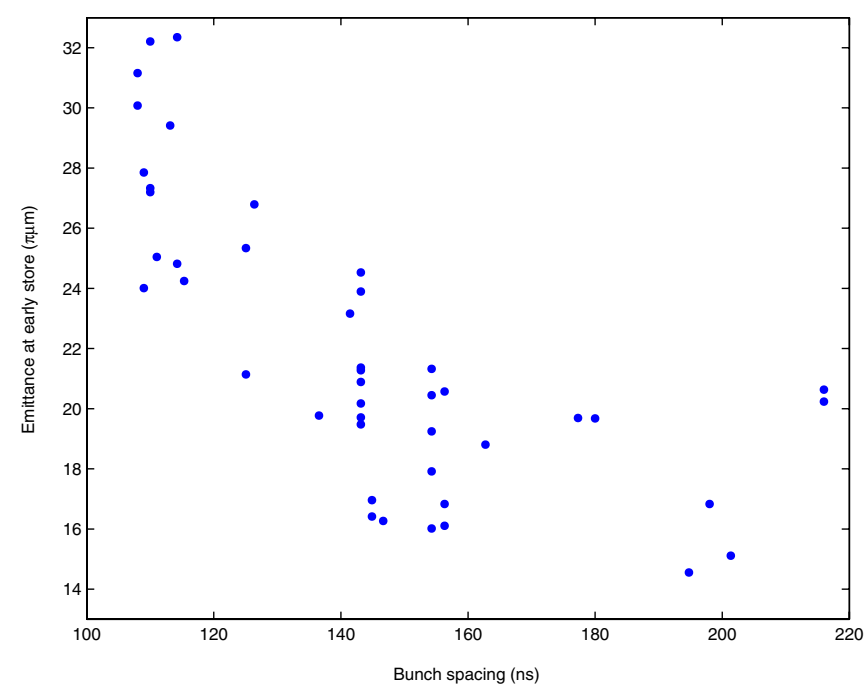

FIG. 6. (Color) Beam emittance at the early store versus the average bunch spacing. The data are from the latest 44 physics fills in run 2005, where the number of bunches injected into RHIC was gradually increased from 55 to 110 . The bunch intensity varied between $0.61 \times 10^{11}$ and $0.91 \times 10^{11}$ protons, not shown.

tons. Note that, regarding to the electron multipacting, the bunch spacing should be more crucial than the bunch intensity, because of the short exponential lifetime of secondary electrons in the bunch gaps.

The beam emittance growth at the store, where the electron cloud is virtually nonexistent, does not show the dependence on the average bunch spacing at all. These data are presented in Fig. 7.

Instead, the beam emittance growth at the store demonstrates a dependence on the beam-beam parameter, defined

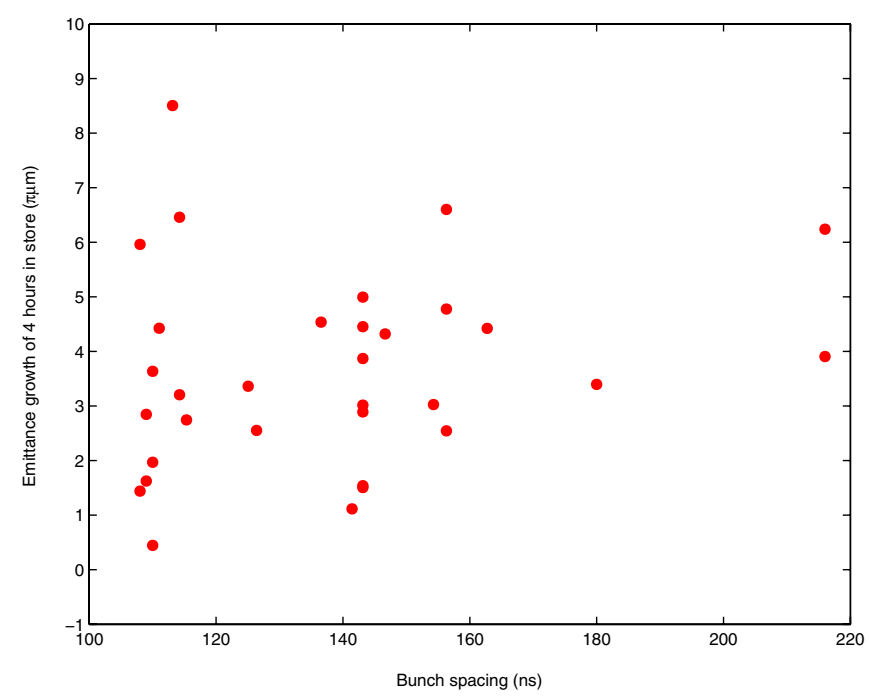

FIG. 7. (Color) Beam emittance growth of 4 hours in store, starting from 1.5 hours after the ramp event, versus the average bunch spacing for the run 2005 fills.
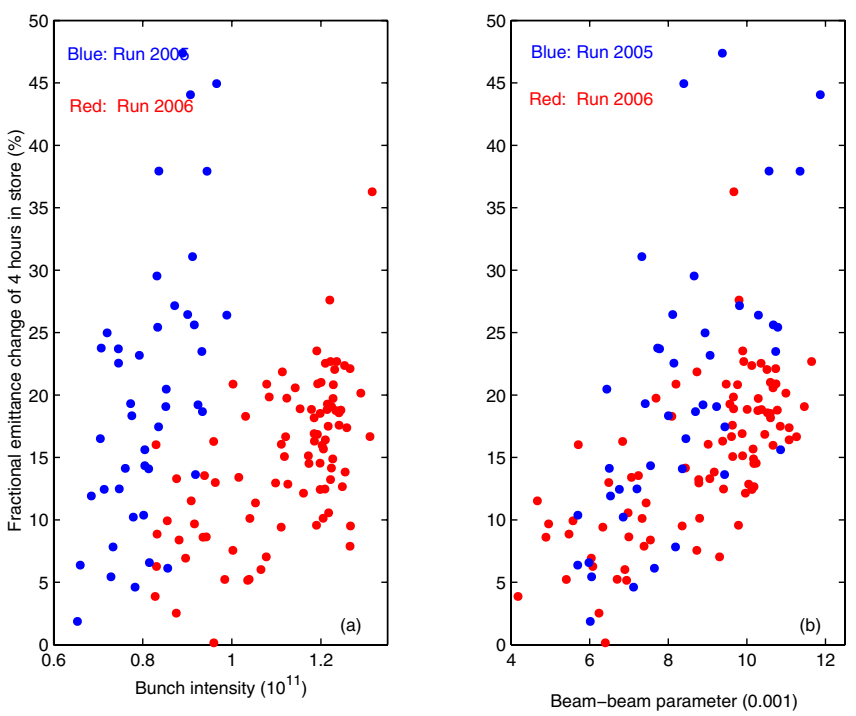

FIG. 8. (Color) The fractional increase of the beam emittance during the first 4 hours of the beam store for runs 2005 and 2006. In (a) it is plotted against the bunch intensity, and in (b) it is plotted against the beam-beam parameter.

as

$$
\xi=3 M N r /(2 \pi \varepsilon)
$$

where $M$ is the number of beam interactions, or collision points, in the ring, $N$ is the bunch intensity, $r$ is the classical proton radius, and $\varepsilon$ is the $95 \%$ normalized beam emittance, with the unit of $\mathrm{m}$. Note that the beam-beam parameter is independent on the number of bunches, and hence is independent on the bunch spacing.

The fractional increase in beam emittance during the first 4 hours of the beam store for run 2005 and run 2006 is plotted against bunch intensity in Fig. 8(a), where it is shown that the bunch intensities in run 2006 are much higher than that in run 2005, but the emittance growth rates are somewhat comparable. In Fig. 8(b) the emittance growth at the store is plotted against the beam-beam parameter. In run 2005 there were 3 collision points, while in run 2006 there were only 2 collision points, the maximum beam-beam parameters achieved in both runs are about the same, i.e., $\xi_{\max }=0.012$. Figure 8 (b) shows clear dependence of the emittance growth on the beam-beam parameter at the store.

\section{B. Dependence on the bunch length}

In run 2006, most of the fills used 110 bunches, thus the bunch spacing was about the same, i.e., $108 \mathrm{~ns}$. The effect of the bunch length on the beam emittance growth was then observed.

Shorter bunches are of great interest from vertex requirements of the physics experiments. Therefore, during run 2006, several attempts were made to reduce the bunch length in RHIC. A main tested technique was a so-called 
longitudinal quadrupole mode pumping at the RHIC injector, AGS, extraction. It aimed to improve the longitudinal phase space matching of the beam during the injection into RHIC, thus achieving a smaller longitudinal emittance and hence a shorter bunch. The technique utilized the excitation of quadrupole mode oscillations in the longitudinal phase space of the beam in AGS and then the extraction of the beam at a correct oscillation phase.

The higher peak current of shorter bunches enhances electron multipactings, and hence enhances the effect of electron cloud on the beam emittance growth. The observation was that, at same total beam intensity, the luminosity of the fills with the quadrupole mode pumping was reduced by $15 \%$. As a typical example, in Fig. 9 two adjacent fills, the normal fill 7856 and the fill 7860 using the quadrupole mode pumping are compared. The beam intensities are at the same level, but the full bunch length of the fill 7856 is $20 \mathrm{~ns}$, while it is only $15 \mathrm{~ns}$ for the fill 7860 . Consequently, the dynamic pressure rise of the fill 7860 is much higher than that of the fill 7856. The beam emittance

Fill 7856
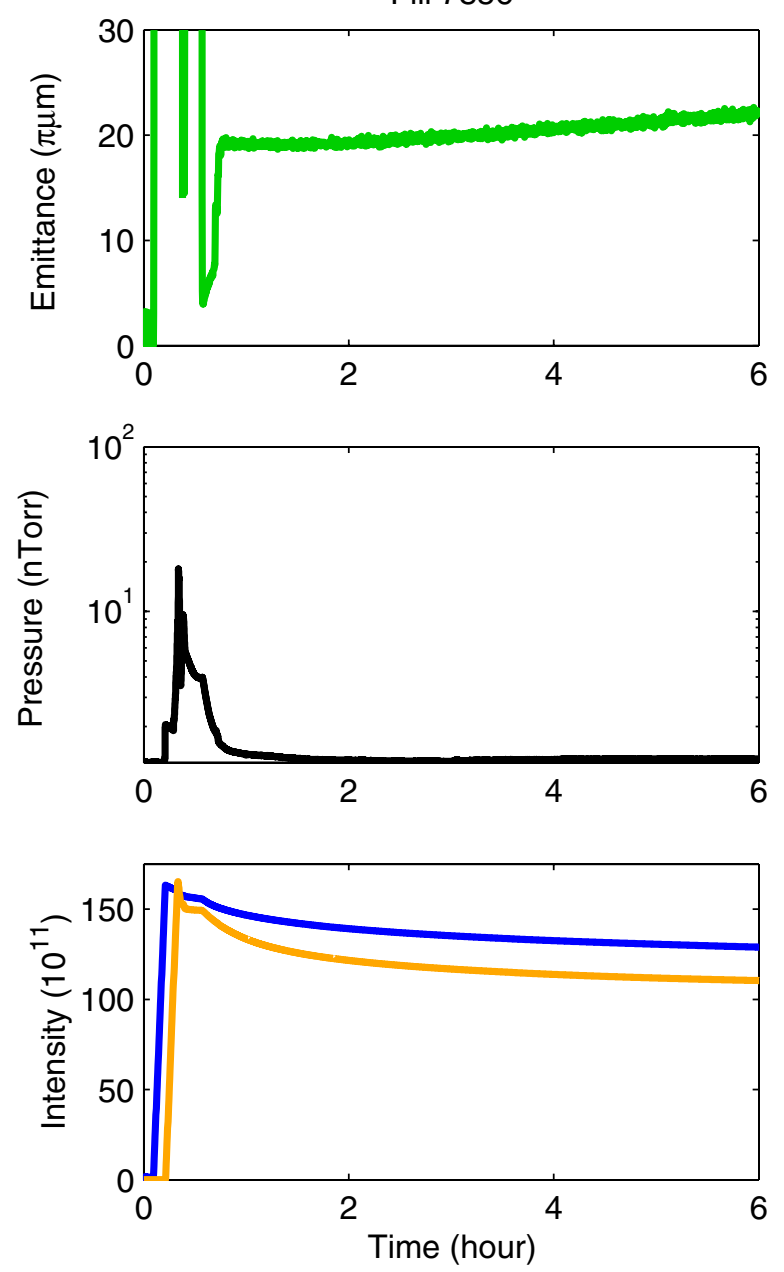

of the fill 7860 at the early store is about $23 \pi \mu \mathrm{m}$, whereas for the fill 7856 it is about $20 \pi \mu \mathrm{m}$. The ZDC coincident rates at the early store are reduced from $9.5 \mathrm{kHz}$ for the fill 7856 to less than $8 \mathrm{kHz}$ for the fill 7860 . The operation with the quadrupole pumping mode had to be abandoned, because of the significantly reduced luminosity.

\section{Dependence on the electron signal from electron detectors}

Because of nonuniform distribution of the electron cloud in RHIC rings, the electron detectors are mainly used to measure the electron multipacting locally in several individual locations. The patterns of the electron multipacting observed in these electron detectors are very similar, and the differences are mainly in magnitudes. Therefore, the correlation of the emittance growth and the electron signals of some electron detectors may reveal qualitatively the emittance growth dependence on the electron cloud.

In Fig. 10, a typical case of the beam bunch train, the fast electron multipacting signal from the electron detector, and
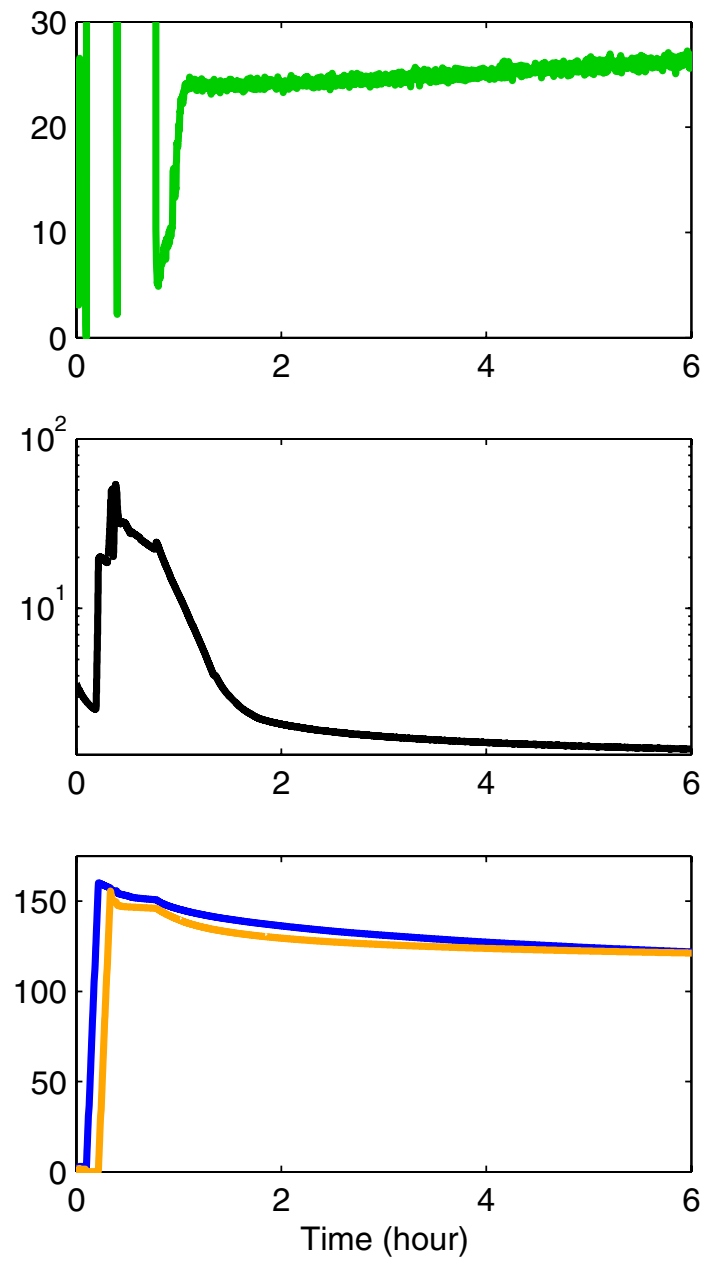

FIG. 9. (Color) The beam intensities, the average pressure rise in 4 worst long straight sections, and the $95 \%$ normalized emittance of the normal fill 7856, and the fill 7860 with the quadrupole mode pumping (shorter bunches). 

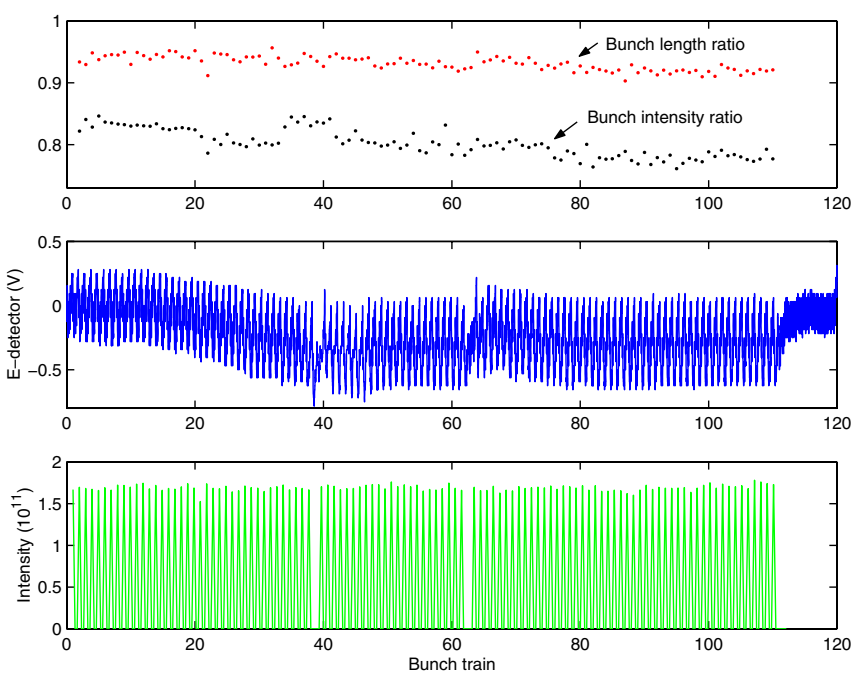

FIG. 10. (Color) Blue bunch train, on the bottom, the electron multipacting signal of the Blue vertical detector at section 1 , in the middle, and the bunch length and bunch intensity reduction ratio along the bunch train, in 20 minutes after the injection is completed.

the bunch length and bunch intensity reduction ratio along the bunch train is shown for an illustration. This is the Blue beam at about 20 minutes after the injection is completed, for the fill 7935. There are two bunch gaps at the buckets 39 and 62 , respectively. The electron signal is from the Blue section 1 vertical detector, and the bunch gaps' effect on the reset of the electron multipacting can be clearly seen. Along with the bunch train, the tail bunches (on the right side) shortened slightly more than the head bunches (on the left side), and bunch intensity reduction along the bunch train is more pronounced. These are agreeable with the CERN SPS observations with the electron cloud [5].
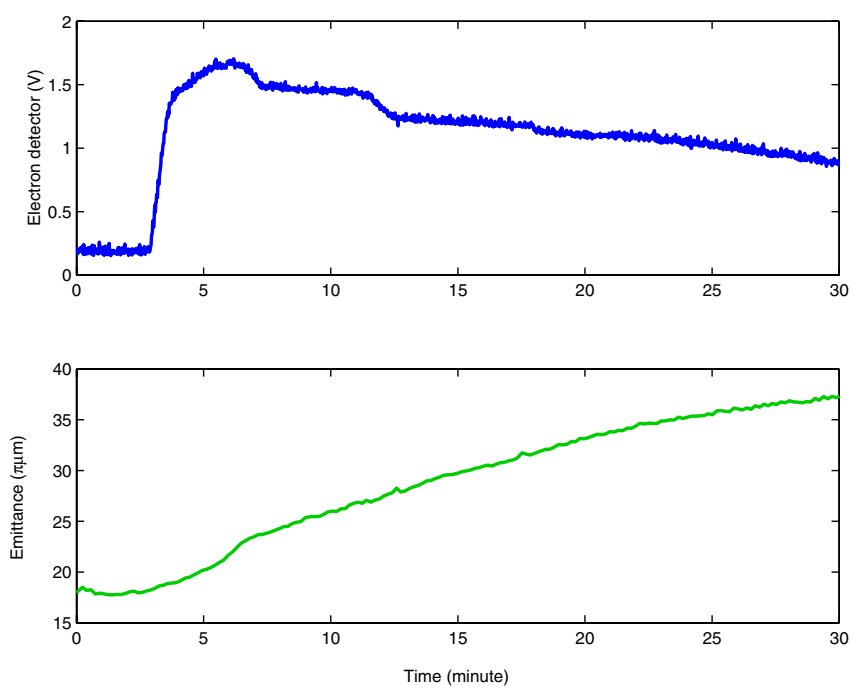

FIG. 11. (Color) Blue ring vertical beam emittance growth measured by the IPM and the slow electron signal from the vertical electron detector at the Blue ring section 1 in RHIC.

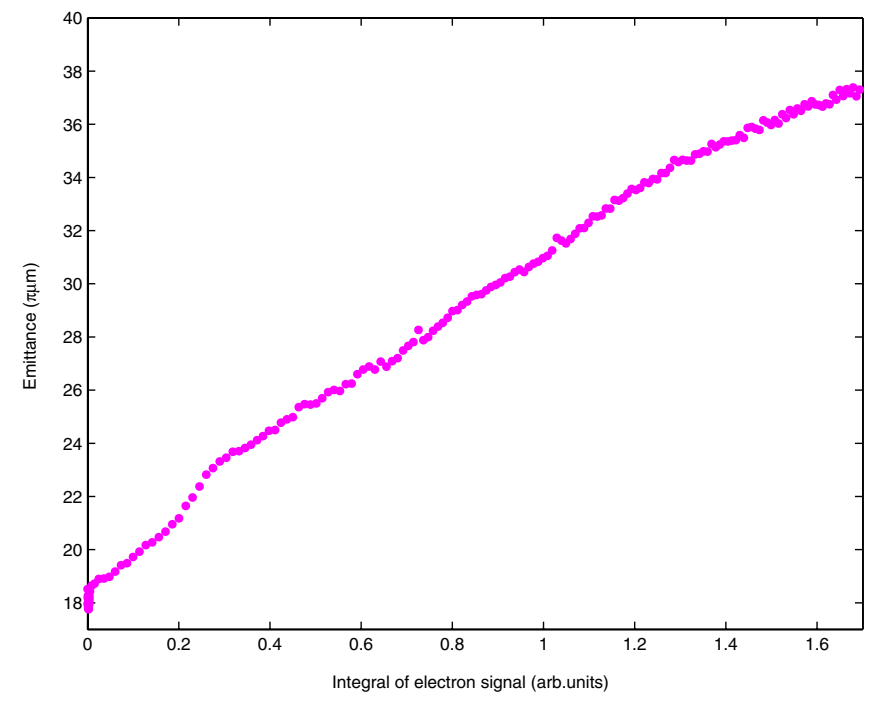

FIG. 12. (Color) The correlation of the Blue ring vertical emittance growth measured by the IPM and the integral of the electron signal detected by the vertical electron detector in the Blue ring section 1 .

To study the correlation of the electron cloud with the beam emittance growth, the slow signal of the electron detector is used. In Fig. 11, the Blue ring vertical emittance growth in the fill 7935, which is shown in Fig. 3, is compared with the slow vertical electron detector signal at the warm section 1 in RHIC. In Fig. 12, the emittance growth is compared with the integral of the electron signal, and the dependence appears to be approximately linear. Similar correlations are observed for other fills as well. It suggests that the accumulated electron kicks on the beam are linearly responsible for the beam emittance growth.

\section{ELECTRON DENSITIES}

The electron cloud at RHIC is nonuniformly distributed around the rings, and this is rather different from other machines having electron cloud problems [4]. For high intensity beam injection and acceleration in RHIC, the dynamic pressure rise at different sections may differ by 4 orders of magnitude, as illustrated in Fig. 2. A total of 12 electron detectors installed in RHIC mainly serve to report the local electron multipacting. The data from those detectors are insufficient for the accurate evaluation of the electron cloud parameters relevant to the beam dynamics, which requires the estimate of the overall electron density in entire rings.

The dynamic pressure rise induced by the electron cloud is monitored around the ring by more than 100 vacuum gauges. Typically there are 3 gauges in a single beam long straight section, and 4 gauges in an interaction region. During the past few years, the serious pressure rise problem in RHIC necessitated numerous beam and bench experiments, which have provided sufficient knowledge regarding the dynamic pumping speed, the species of the 
TABLE IV. Electron density of the fill 7935 in 4 Yellow straight sections and 1 interaction region section. These 5 sections contain about $90 \%$ of all electrons in the entire ring, which are responsible for the Yellow beam emittance growth.

\begin{tabular}{cccccc}
\hline \hline Location & Yo1 & Yo4 & Yi7 & Yo9 & IP4 \\
\hline E-density, $10^{10} / \mathrm{m}^{3}$ & 15.8 & 17.3 & 20.9 & 27.4 & 37.2 \\
\hline \hline
\end{tabular}

molecules, the chamber surface properties and the related electron desorption rates, etc. [2,3].

Secondary electrons are generated during bunch passages, then subside in bunch gaps. At the passage of each bunch, all electrons would hit the wall with the welldefined energy gained from the passing bunch. It results in the production of gas molecules due to electron desorptions, which can be satisfactorily accounted using the vacuum gauges at the equilibrium conditions. In fact, to calculate the secondary electrons using the resulted pressure rise might be one of the best measurements of the electron density generated in each section.

In Table IV, the electron density of the fill 7935 is shown, where the beam emittance growth has a rate of $40 \pi \mu \mathrm{m}$ per hour. The data for 4 Yellow long straight sections and 1 interaction region section are shown. These 5 sections contain about $90 \%$ of all electrons in the entire ring, so they might be most responsible for the Yellow beam emittance growth. Under equilibrium vacuum pressure conditions the number of electrons are evaluated. Then with the assumption of the uniform distribution of the electrons before the bunch arrival, the electron densities in the beam pipes are calculated.

The total length of these five sections is $153 \mathrm{~m}$, whereas the entire ring circumference is $3834 \mathrm{~m}$. The average electron density in the entire ring, therefore, would be about $1.7 \times 10^{10} / \mathrm{m}^{3}$ for the Yellow beam in the fill 7935. For other machines coping with the electron cloud problems, such as CERN SPS [4,5] and B-factories [6,7], the distribution of the electron multipacting in the rings is more uniform, and the usual electron density would be more than 10 times higher, i.e., larger than $10^{11} / \mathrm{m}^{3}$. At RHIC, strong electron multipacting takes place at only a few locations, as the result the average electron density in the entire ring is low. Note that, under RHIC operation conditions, with lower beam intensities than on the fill 7935, the average electron density is even lower, in the order of $10^{9} / \mathrm{m}^{3}$.

\section{SUMMARY AND DISCUSSION}

With the significantly improved beam intensities in RHIC polarized proton runs, the beam emittance growth during the injection and acceleration becomes a luminosity limit. The emittance growth has been found to depend on the dynamic pressure rise. The direct effect of the pressure rise on the beam, i.e., the beam-gas effect, cannot explain the emittance growth. On the other hand, since the dynamic pressure rise at RHIC proton operations is caused by the electron cloud, the electron-beam interaction during the bunch passage is likely causing the emittance growth.

The electron cloud induced beam emittance growth has limited the machine luminosity at KEKB and PEPII [6,7], where the emittance growth dependence on the beam intensity is similar to that observed at the RHIC. These include the existence of the intensity threshold of the emittance growth, and the linear beam size growth above the threshold at high intensities.

An important feature of the emittance growth at RHIC is that it is not accompanied by a beam instability. Therefore, the emittance growth below the instability threshold has attracted attention and currently it is under intense study. Simulations suggest that the electron pinch causes a nonlinear incoherent tune shift along the bunch and the excited resonance might be the source of the emittance growth [8].

The beam emittance growth at RHIC, which is the focus of this paper, has much slower growth rates than that due to the beam instabilities. For example, in run 2006, the machine tuning efforts in the latest 6 weeks focused on the beam emittance growth with a rate of $2 \pi \mu \mathrm{m}$ per hour. The emittance growth was small; nevertheless, it had a significant effect on the machine luminosity, of order $12 \%$.

It is fortunate that the electron cloud is absent at the RHIC store. The beam-beam tune related emittance growth at RHIC proton beam store induces a typical 3\% growth per hour, and hence a 15 hour store is possible. Given the emittance growth observed at the injection and acceleration (depended on the pressure rise), the growth rate would be 4 times higher, i.e., about $12 \%$ per hour, and only about 4 hours of an optimized store would be possible.

The measurement of the beam emittance growth at such a slow rate is very difficult. In fact, at RHIC a consistent beam emittance study can only be made possible by using the ZDC coincidence at the beam collisions. In beam experiments, we intentionally used much higher beam intensity to get a faster emittance growth, so that the emittance measurement using conventional instruments becomes possible.

Accompanied with this faster emittance growth rate, the average electron density in the two RHIC rings is about $10^{10} / \mathrm{m}^{3}$. Note that in RHIC, strong electron multipacting only shows up at a few sections; consequently the electron distribution in both rings is very nonuniform, and the average electron density over the entire ring is low. This relative low electron density may explain the slow emittance growth rate at RHIC.

\section{ACKNOWLEDGMENTS}

This work was supported by Brookhaven Science Associates, LLC under Contract No. DE-AC0298CH10886 with the U.S. Department of Energy and by a sponsored research grant from Renaissance Technologies 
Corporation.

[1] S. Y. Zhang, H.C. Hseuh, W. Fischer, H. Huang, and T. Roser, BNL Report No. C-A/AP/220, 2005.

[2] S. Y. Zhang et al., Proceedings of Particle Accelerator Conference, Knoxville, TN, 2005 (IEEE, New York, 2005), p. 4308.

[3] W. Fischer et al., Proceedings of Particle Accelerator Conference, Albuquerque, NM, 2007 (IEEE, New York, 2007), p. 759.
[4] F. Zimmermann, Proceedings of the Particle Accelerator Conference, Chicago, IL, 2001 (IEEE, New York, 2001), p. 666.

[5] G. Arduini et al., Proceedings of ECLOUD'04, 31st Advanced ICFA Beam Dynamics Workshop on Electron-Cloud Effects, Napa, CA [Report No. CERN2005-001, CARE-Conf-05-001-HHH, LBNL-56372, SNS-104000000-TR0024-R00, pp. 31-47 (2005)].

[6] H. Fukuma, Ref. [5], pp. 15-19.

[7] A. Kulikov et al., Ref. [5], p. 21-24.

[8] E. Benedetto, G. Franchetti, and F. Zimmermann, Phys. Rev. Lett. 97, 034801 (2006). 